\title{
Building nanoparticles using diamondoids structures as a carrier of the drug: Density functional theory study
}

\author{
Huda M. Jawad
}

Department of Physics, College of Science, University of Al-Mustansiriyah, Baghdad, Iraq.

Corresponding author(s) E-mail: drhuda293@gmail.com

Key Words: Diamondods, Aluminum phosphide, Density functional theory

\section{Abstract}

Building nanoparticles of Aluminum phosphide (AlP) have been prepared by Density functional theory method. These particles have been built on the diamondoids structures by raising carbon atoms and put atoms equivalent atomic number. Atomic number for Aluminum is (13) and Phosphorus is (15). It can be used these structures to the drug delivery .Depend on electronic structure and vibration properties of AlP nanocrystal. In order to full investigate, gap energy, electrostatic potential, density of states, tetrahedral angle, dihedral angle, bond length, IR intensity, Raman spectrum. The results show that AlP diamondoids are nano-particles, structural properties as close as possible to those of bulk zincblende structure. The values of the energy gap diamantine is $(3.5 \mathrm{eV})$ with respect to the bulk value $(2.5 \mathrm{eV})$. The green color signifies the neutral electrostatic potential. This means that diamantane insulating material and this helps us in the bonding process with the drug without that interact with any medication is delivery medicine to the affected places. Dihedral and tetrahedral angles in AlP-diamantane near ideal bulk zincblende value of this angle values reflect the stability of diamondoids structures which is useful for our study to get the inert and no harm structures after bonding there with different drugs to use it as drugs carriers. The bond lengths in AlP-diamantane found at $2.38 \AA$ and the experimental value of AlP bulk bond length at $2.293 \AA$. IR intensity of diamondoids divided into two regions depending on the properties of vibration or the gap separation them and Raman spectrum active. UV Visible spectrum of diamondoids structure the Excitation energy equal to $(1.8849 \mathrm{eV})$, the wave length Absorption is (657.79 nm). 


\section{1-Introduction}

Aluminum phosphide is inorganic compound with the chemical formula AlP used as a wide band gap semiconductor. The energy gap equal to $2.5 \mathrm{eV}$ indirect. AlP crystals are dark grey to dark yellow in color and have a zincblende crystal structure [1]. Industrially, AlP is a semiconductor material that is usually alloyed with other binary materials for applications in devices such as light-emitting diodes (e.g. aluminum gallium indium phosphide) [2]. Diamondoid molecules are cagelike, ultra-stable, saturated

hydrocarbons. These molecules are ringed compounds, which have a diamond-like structure consisting of a number of six-member carbon rings fused together [3]. The basic repetitive unit of the diamondoids is a ten-carbon tetracyclic cage system called adamantine. In this study used dimantane with chemical formula $\left(\mathrm{C}_{14} \mathrm{H}_{20}\right)$ (Figure 1) show the geometrically optimized of AlP dimantane,Apraclonidine and AlP dimantane bonded with Apraclonidine [4]. The ability to build complex diamondoid medical nanorobots (Freitas 1998, 2000a, 2005a, 2006, 2007) to molecular precision, and then to build them cheaply enough in sufficiently large numbers to be useful therapeutically will revolutionize the practice of medicine (Freitas 2008) and surgery (Freitas 2005b). The first theoretical design study of a complete medical nanorobot ever published in a peer-reviewed journal described a hypothetical artificial mechanical red blood cell or "respirocyte" made of 18 billion precisely arranged structural atoms (Freitas 1998). The respirocyte would be a bloodborne spherical 1-micron diamondoid 1000. This nanorobot would deliver 236 times more oxygen to body tissues per unit volume than natural red cells [5].In this study can be used diamandoids as a carrier for the drug. It can be bonded the apraclonidine with chemical formula $\left(\mathrm{C}_{9} \mathrm{H}_{10} \mathrm{Cl}_{2} \mathrm{~N}_{4}\right)$ drug with dimantane, where apraclonidine Solution contains apraclonidine hydrochloride, an alpha adrenergic agonist, in a sterile isotonic solution for topical application to the eye. It has the action of reducing intraocular pressure [6]. 

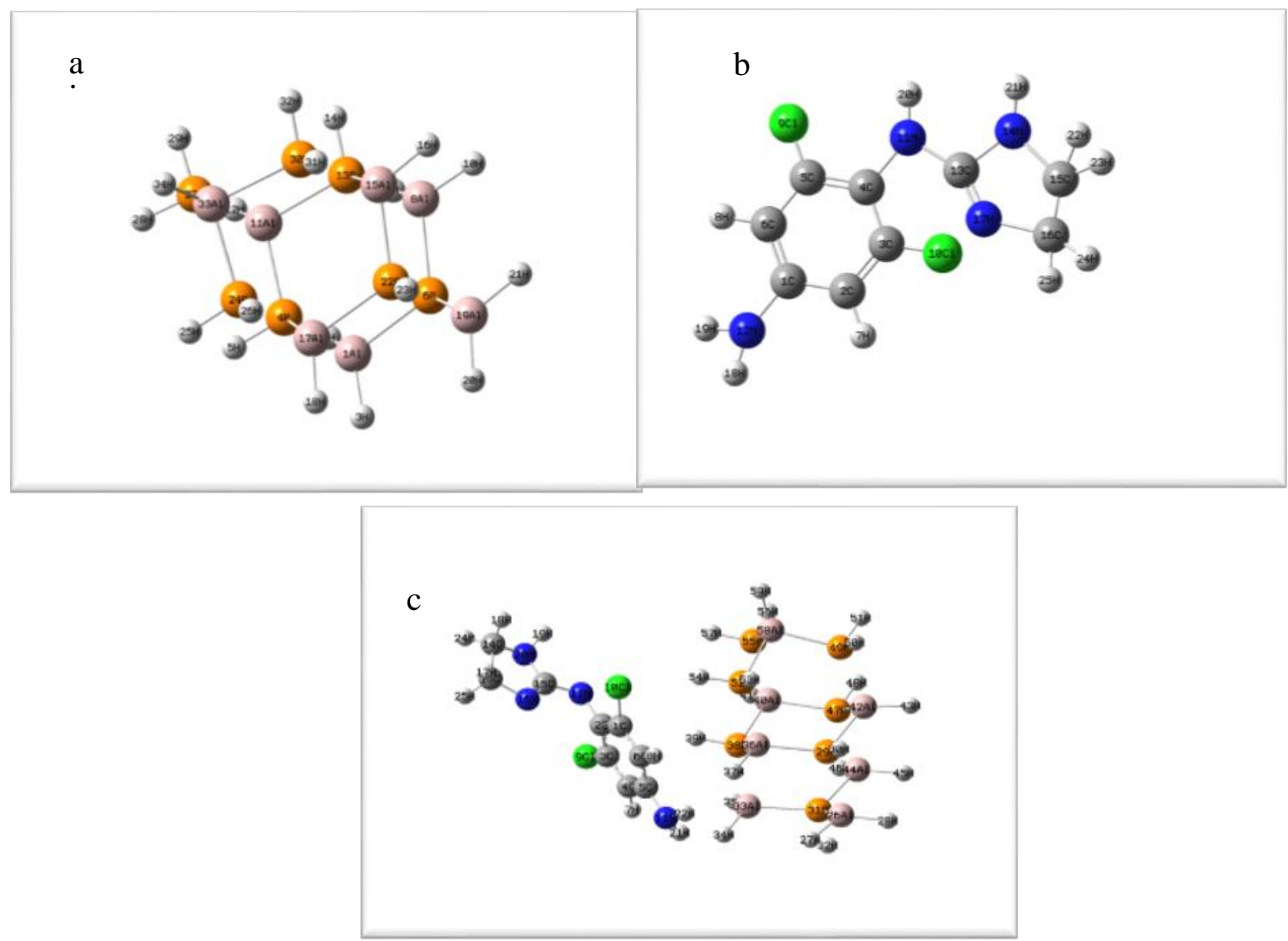

Figure (1). Geometrically optimized a- AlP-diamantane $\left(\mathrm{Al}_{7} \mathrm{P}_{7} \mathrm{H}_{20}\right)$, b- Apraclonidine $\left(\mathrm{C}_{9} \mathrm{H}_{10} \mathrm{Cl}_{2} \mathrm{~N}_{4}\right)$

\section{2- Theory} and c- AlP-diamantane bonded with Apraclonidine.

By the early 21st century, this deep molecular familiarity with the human body along with continuing nanotechnological engineering advances, has set the stage for a shift from present-day molecular scientific medicine in which fundamental new discoveries are constantly being made, to a future molecular technologic medicine in which the molecular basis of life, by then well-known, is manipulated to produce specific desired results. The comprehensive knowledge of human molecular structure so painstakingly acquired during the previous century will be extended and employed in this century to design medically-active microscopic machines. These machines, rather than being tasked primarily with voyages of pure discovery, will instead most often be sent on missions of cellular inspection, repair and reconstruction. The principal focus will shift from medical science to medical engineering Nanomedicine (Freitas 1999, 2003) will involve designing and building a vast proliferation of incredibly efficacious molecular devices, and then deploying these devices in patients to establish and maintain a continuous state of human healthiness " Physicians aim to make tissues healthy," wrote one early pioneer (Drexler 1986) [7]. The geometrically optimized of AlP diamantine with 
chemical formula $\left(\mathrm{Al}_{7} \mathrm{P}_{7} \mathrm{H}_{20}\right)$, using density functional theory at the generalized gradient approximation of Perdew, Burke and Ernzerhof (PBE) /6-31g (d) basis set which included polarization functions . This created using Gaussian 09 program auxiliary by Gaussian view 5.08. We will review some of the fundamental aspects of electronic structure theory in order to lay the foundations for the theoretical discussion on density functional theory (DFT). The ultimate goal of most quantum chemical approaches is the - approximate - solution of the time-independent, nonrelativistic Schrödinger equation [8]. In this work has been to bond diamantae with Apraclonidine by van der Waals force. In physical chemistry, the van der Waals forces (or van der Waals interaction), named after Dutch scientist Johannes Diderik van der Waals, are the residual attractive or repulsive forces between molecules or atomic groups that do not arise from covalent bonds, nor ionic bonds.It can be shown that van der Waals forces are of the same origin as the Casimir effect, arising from quantum interactions with the zero-point field. The resulting van der Waals forces can be attractive or repulsive [9].

\section{Results and discussion}

Figs. (2-a, 2-b and 2-c) Show the distribution of bond lengths in AlP-diamondoids. The dashed line represents the experimental value of AlP bulk bond length at 2.293 $\AA$ [10]. Shows the distribution of bond lengths in AlPdiamantane, Apraclonidine and bond the apraclonidine with AlP diamantane. The main reason for the nonequivalent bond lengths in our case is the location of atoms either near or far from the surface, and their bonding with either one or two $\mathrm{H}$ atoms. The fig (2-a) starts with a sharp, high value for the number of phosphorus-hydrogen bonds. The phosphorus-hydrogen bonds are the shortest bonds in the present molecules. P-H, $\mathrm{P}-2 \mathrm{H}, \mathrm{Al}-\mathrm{H}$ and $\mathrm{Al}-2 \mathrm{H}$ refer to the bonding of a phosphorus and Aluminum atoms with one or two surface hydrogen atoms respectively. This fig. does not distinguish between the two cases $\mathrm{P}-\mathrm{H}, \mathrm{P}-2 \mathrm{H}, \mathrm{Al}-\mathrm{H}$ and $\mathrm{Al}-2 \mathrm{H}$. The distribution of Al-Pdiamantane nearly centred on the experimental value (dashed line in Fig. (2-a)) [11].At fig. (2-b) Show the distribution of bond lengths for Apraclonidine. High value for the number of carbon-hydrogen bonds. The nitrogen-hydrogen bonds are the shortest bonds in the present molecules. At fig. (2-c) show AlP diamantine bonded with Apraclonidine 

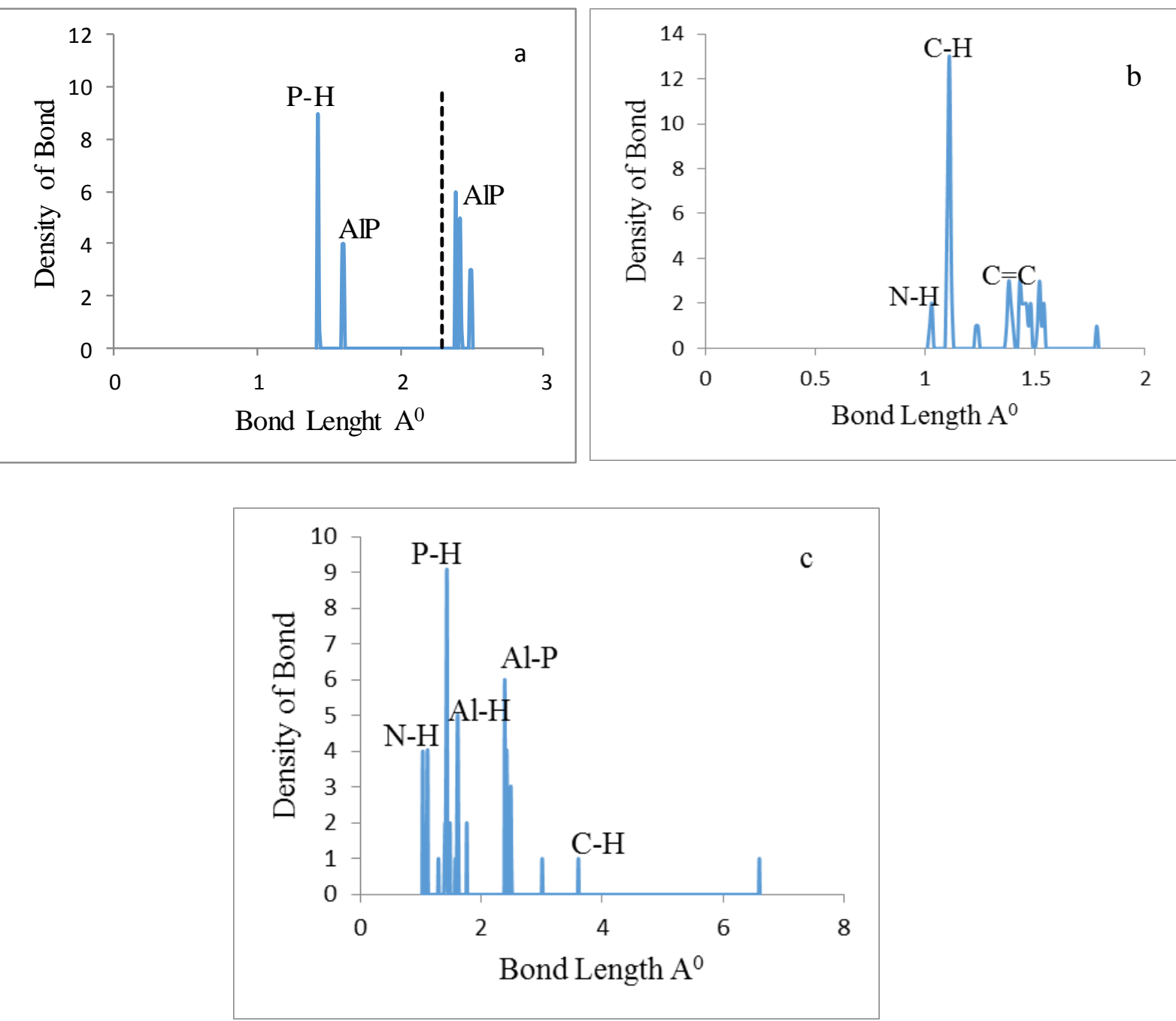

Figure (2). a- Distribution of bond lengths in AlP-diamantane, b- Apraclonidine and c- AlPdiamantane bonded with Apraclonidine. The dashed line represents the experimental value of AlP bulk bond length.

Figs. (3-a, 3-b and 3-c) Show the density of energy states of geometrically optimized AlP-diamantane, Apraclonidine and AlP-diamantane bonded with Apraclonidine as a function of energy level. Fig. (3-a) the values of the energy gap diamantine is $(3.5 \mathrm{eV})$ with respect to the bulk value $(2.5 \mathrm{eV})$ as expected in this range. The near discrete levels of diamantine. Fig. (3-b) the values of the energy gap (3.4 eV) near-continuous levels of Apraclonidine. Fig. (3-c) show the density of energy states of geometrically optimized AlP-diamantane bonded with Apraclonidine as a function of energy level [12]. 

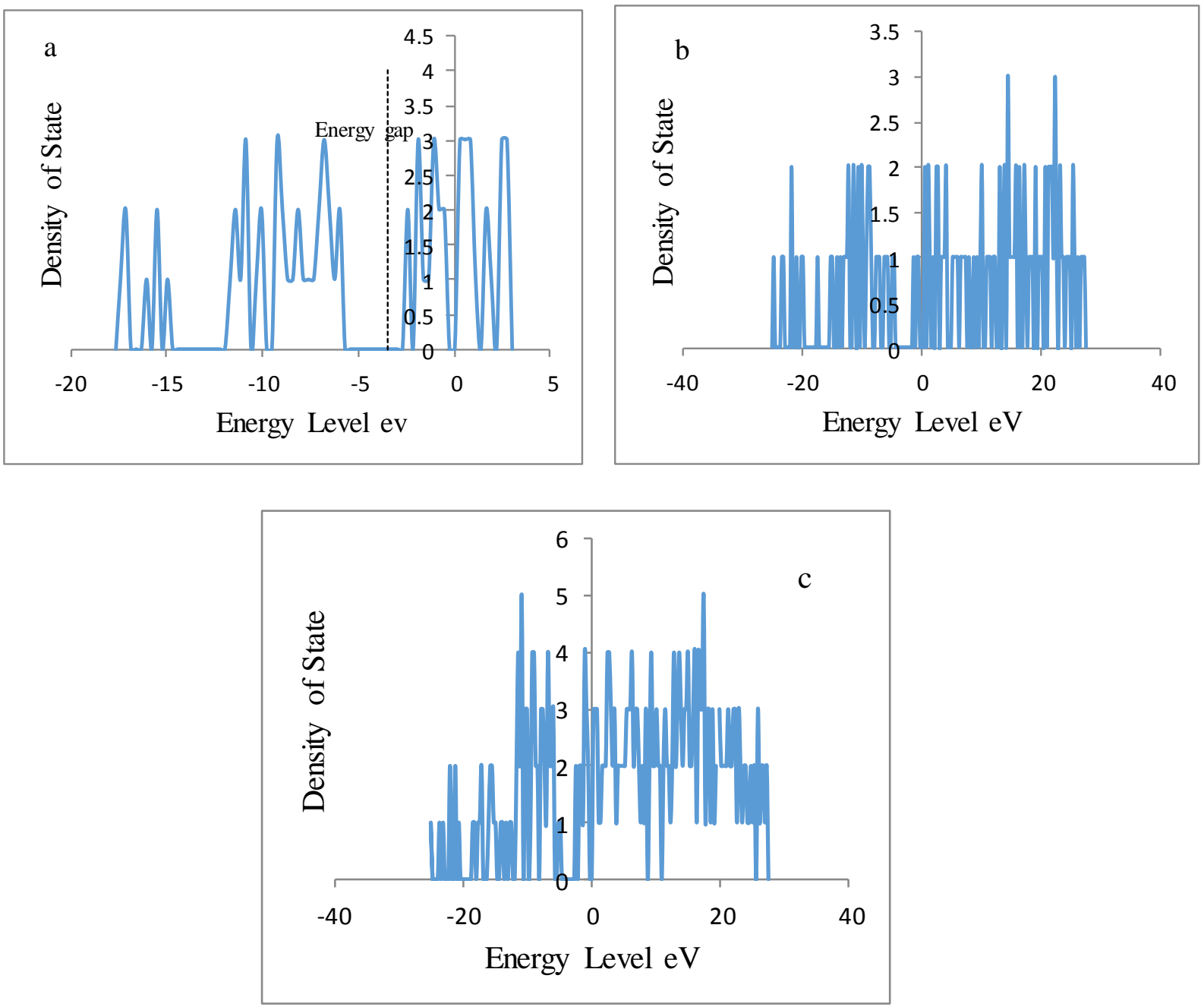

Figure (3). Density of energy states of geometrically optimized (a) AlP-diamantane and (b) Apraclonidine and c- AlP-diamantane bonded with Apraclonidine. The dotted line represents the Fermi level. 
Figs. (4-a, 4-b and 4-c) Show the distribution of tetrahedral angle the values of AlP diamantane, Apraclonidine and AlP-diamantane bonded with Apraclonidine angles.Fig. (4-a) the ideal bulk zincblende value of this angle at $109.47^{\circ}$ is shown [12]. The distribution shows a narrow range around this angle $\left(109.47^{\circ} \pm 13^{\circ}\right)$, with the highest peak shifted one or two degrees from this value in AlP diamantane. Surface relaxation is responsible for this distribution, as it leads to the deviation of these angles from their ideal value. Fig. (4-b) is shown with the peak shifted one or two degrees from this value in Apraclonidine. Fig (4-c) note and AlP-diamantane bonded with Apraclonidine tetrahedral angles.
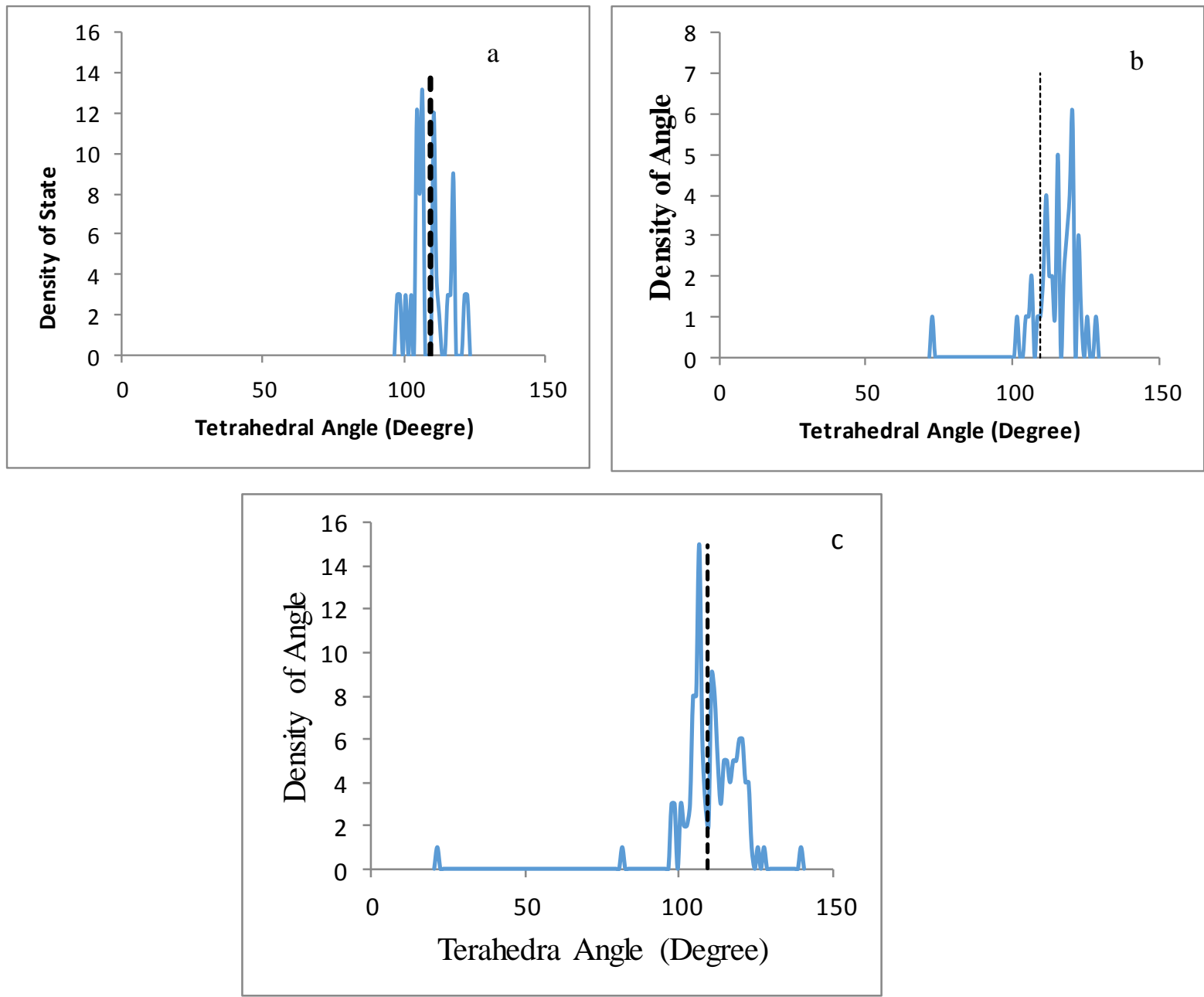

Figure (4). Distribution of the values of (a) AlP-diamantane and (b) Apraclonidine (c) AlPdiamantane bonded with Apraclonidine tetrahedral angles. The dashed line represents the ideal bulk zincblende value of this angle, at $109.47^{\circ}$ [12]. 
Figs. (5-a, 5-b and 5-c) Show of AlPdiamantane, Apraclonidine and AlPdiamantane bonded with Apraclonidine the dihedral angle. The ideal bulk zincblende values of these angles are \pm 60 and \pm 180 [12]. Again, surface relaxation is responsible for the narrow deviation from this ideal values. Fig. (5-a) The distribution dihedral angle of AlP-diamantane. Fig. (5-b) Apraclonidine dihedral angle. Fig. (5-c) AlP-diamantane bonded with Apraclonidine the dihedral angle.
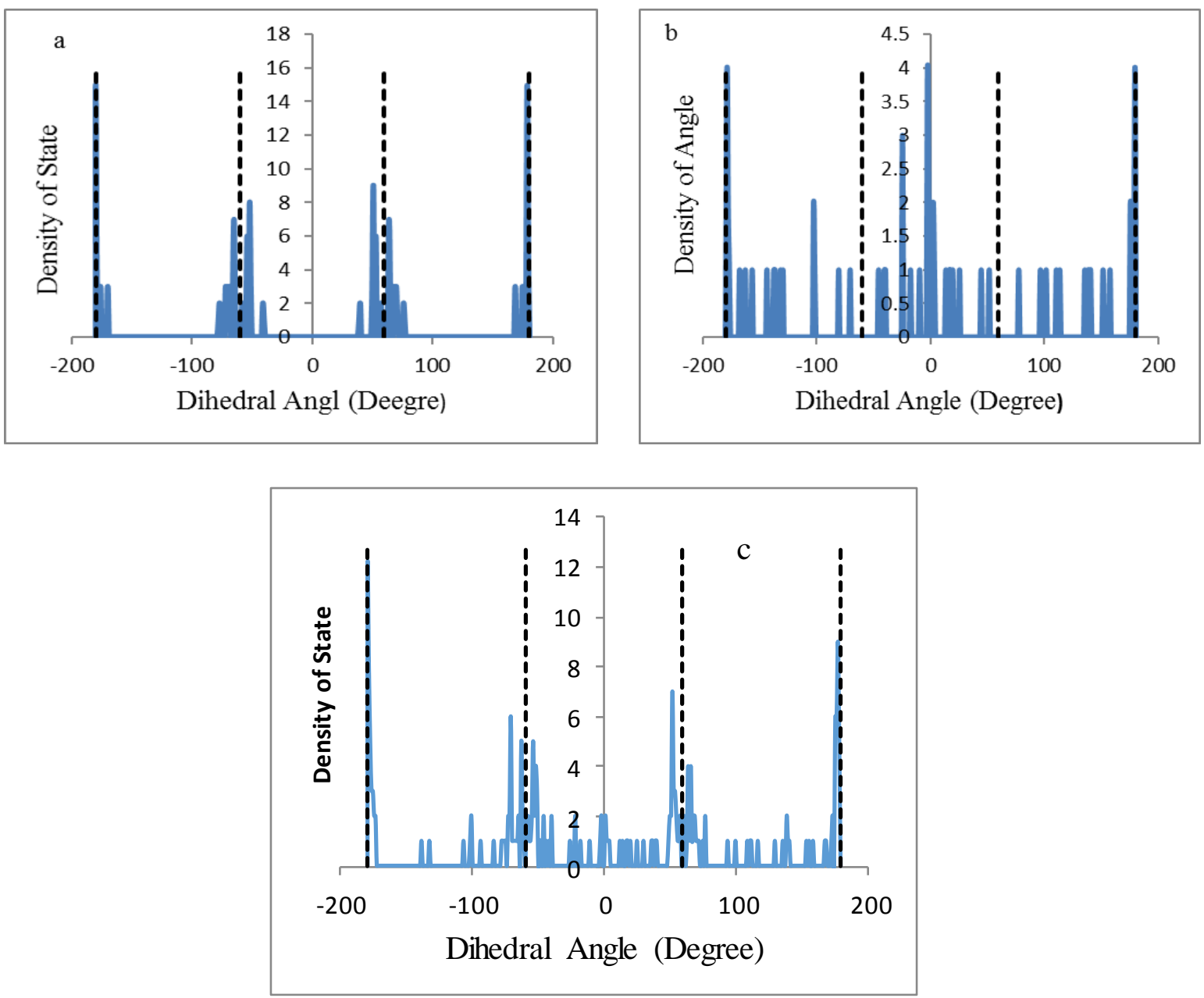

Figure (5). Distribution of (a) AlP-diamantane, (b) Apraclonidine and (c) AlP-diamantane bonded with Apraclonidine dihedral angles. The dashed lines represent the ideal values of zincblende structure, at $-180^{\circ},-60^{\circ}, 60^{\circ}$ and $180^{\circ}$ [13]. 
Figs. (6-a, 6-b and 6-c) show the electrostatic potential of diamondoids structure molecules using PBE/6-31G (d) basis sets, Apraclonidine and AlP diamantane bonded with Apraclonidine. The molecular electrostatic potential (MEP) mapping is very useful in the investigation of the molecular structure with its physiochemical property relationships.A portion of the molecule that has a negative electrostatic potential is susceptible to electrophilic attack. The red and blue regions in the MEP map refer to the regions of negative and positive potentials and correspond to the electron-rich and electron-deficient regions respectively whereas the green color signifies the neutral electrostatic potential, and the color spectrum is mapped to all other values by linear interpolation [13].Fig. (6-a) Alp diamantane electrostatic potential (MEP) mapping the green color signifies the neutral electrostatic potential. Fig. (6-b) show Apraclonidine electrostatic potential has been rich regions of electrons has a red color and poor regions of electrons, a blue color. Fig. (6-c) refers Alp diamantane bonded with Apraclonidine electrostatic potential.

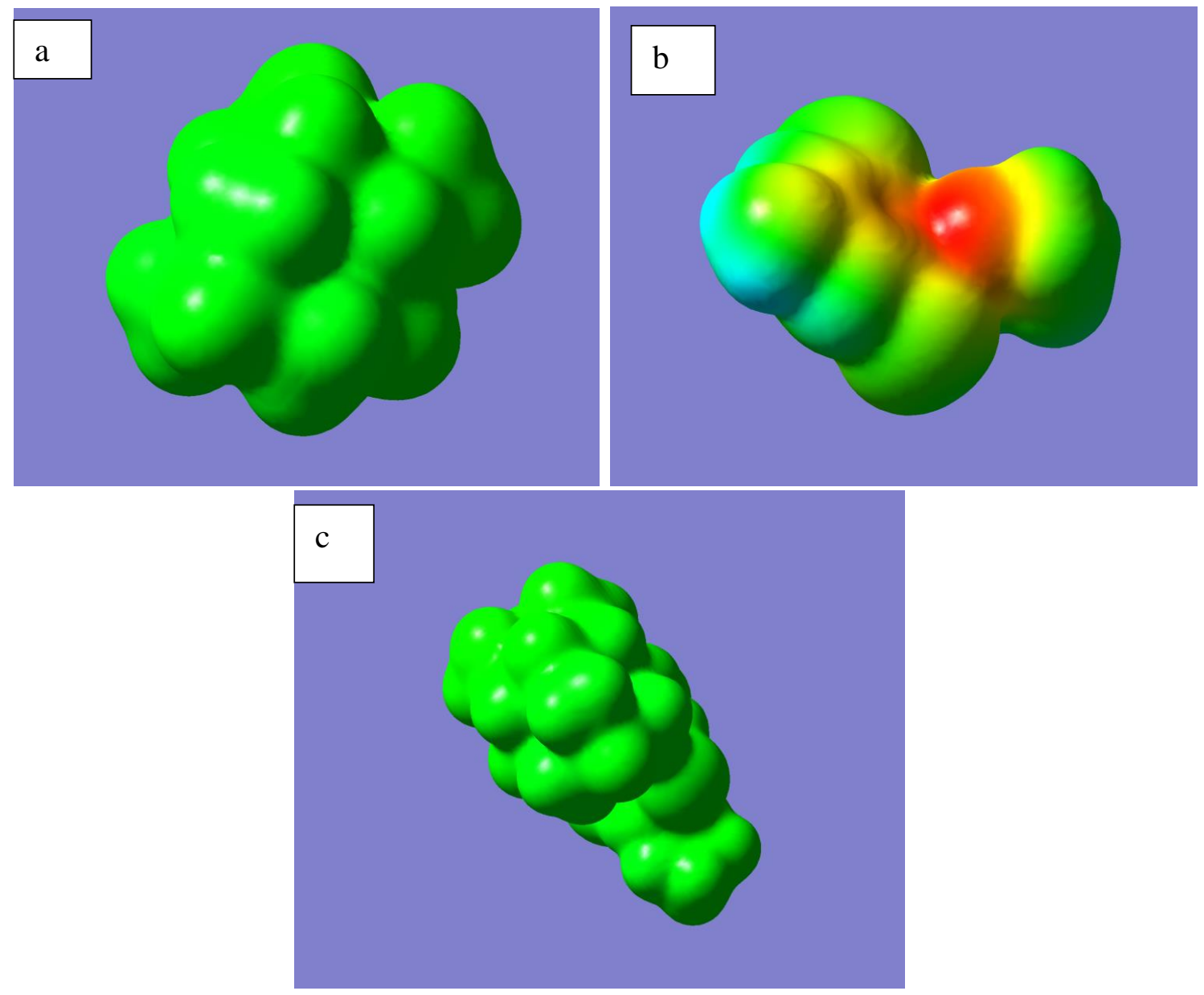

Figure (6). Electrostatic potential of of (a) AlP-diamantane, (b) Apraclonidine and (c) AlPdiamantane bonded with Apraclonidine 
Figs. (7-a, 7-b and 7-c) show IR intensity of diamondoids structure molecules using PBE/6-31G (d) basis sets, Apraclonidine and AlP diamantane bonded with Apraclonidine. Fig. (7-a) IR spectrum of AlP diamondoids structure molecules diamantane, which can be divided into two regions depending on the properties of vibration or the gap separation them. In first region, results show $(0-300) \mathrm{cm}^{-1}$ region is characterized by pure AlP and The region around the broad peak at $650 \mathrm{~cm}^{-1}$ has Al-P-H. This includes the $1850 \mathrm{~cm}^{-1}$ mode in $\mathrm{Al}-\mathrm{H}$ and $\mathrm{P}-\mathrm{H}$ vibrations include stretching (symmetric and asymmetric) and the bending mode of vibration (wagging, scissoring, rocking and twisting). Figure (7-b) note IR intensity of Apraclonidine and Figure (7-c) show IR intensity of AlP-diamantane bonded with Apraclonidine.

IR Spectrum

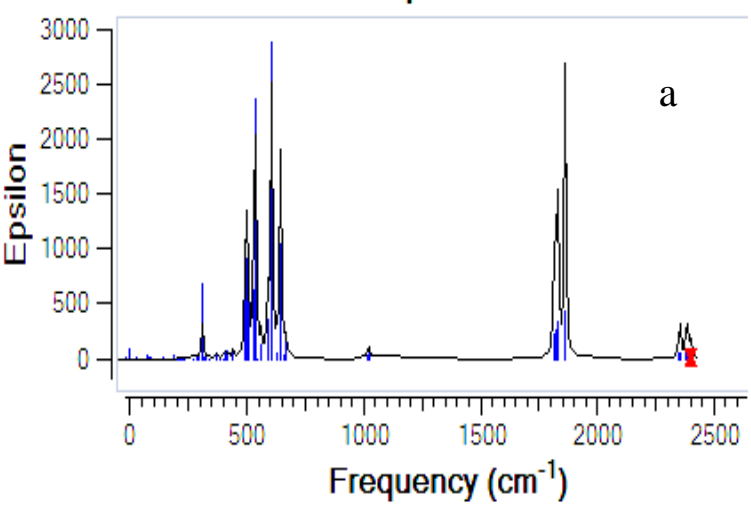

IR Spectrum

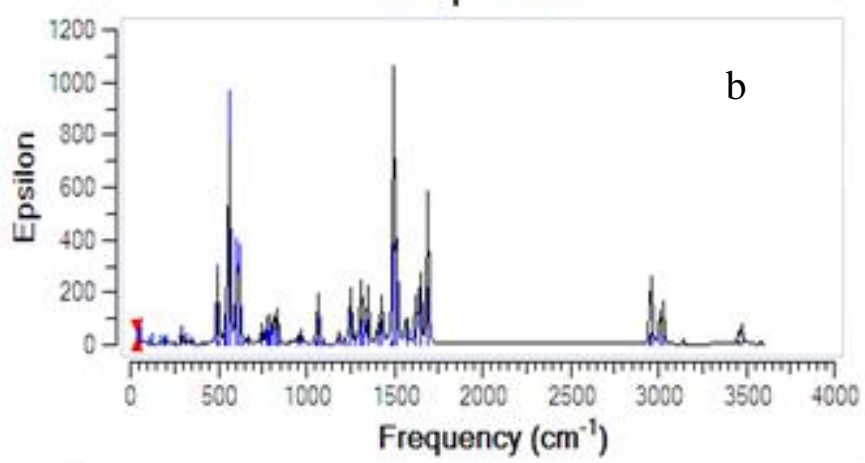

IR Spectrum

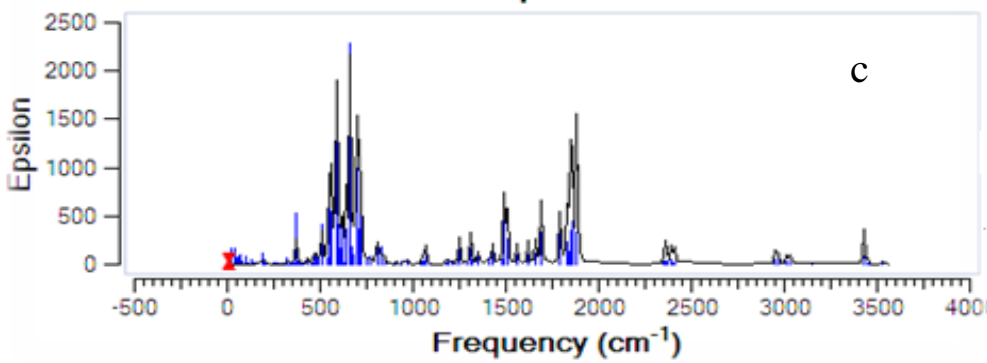

Figure (7). IR intensity of (a) AlP-diamantane, (b) Apraclonidine and (c) AlP-diamantane bonded with Apraclonidine 
Figs. (8-a, 8-b and 8-c) show Raman spectrum of diamondoids structure, Apraclonidine and AlP diamantane bonded with Apraclonidine. At fig. (8-a) the Raman spectrum of AlP diamondoids structure molecule. It can be shown from this figure that the active region in IR is within less activity in Raman and this is due to different in selection rule as the different in intensity between IR and Raman for the same frequency and symmetry. At figure (8-b) the Raman spectrum of Apraclonidine and figure (8-c) the Raman spectrum of AlP diamantane bonded with Apraclonidine.
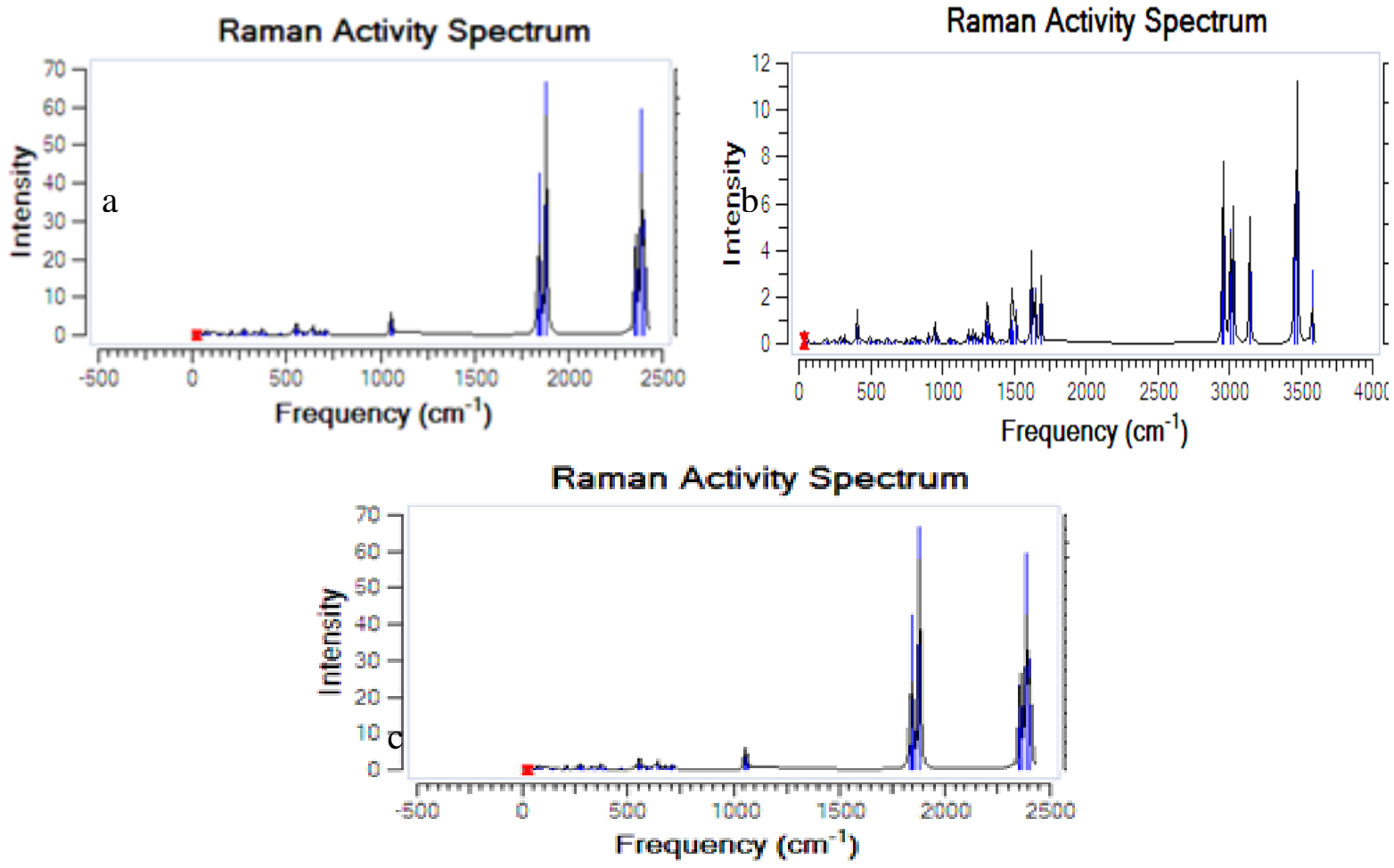

Figure (8). Raman spectrum of (a) AlP-diamantane, (b) Apraclonidine and (c) AlPdiamantane bonded with Apraclonidine

Figures (9-a and 9-b) show UV Visible spectrum of diamondoids structure and Apraclonidine. Fig. (9-a) note the Excitation energy equal to $(1.8849 \mathrm{eV})$, the wave length Absorption is $(657.79 \mathrm{~nm})$ and frequency is (0.0002). Fig. (9-b) show UV Visible spectrum of Apraclonidine Excitation energy equal to $(0.9187 \mathrm{eV})$, the wave length Absorption is $(1349.53 \mathrm{~nm})$ and frequency is $(0.0024)$. 
UV-VIS Spectrum

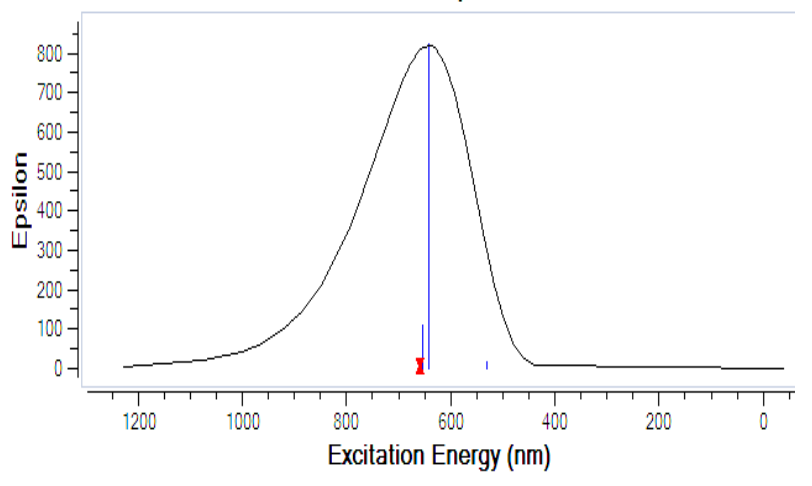

UV-VIS Spectrum

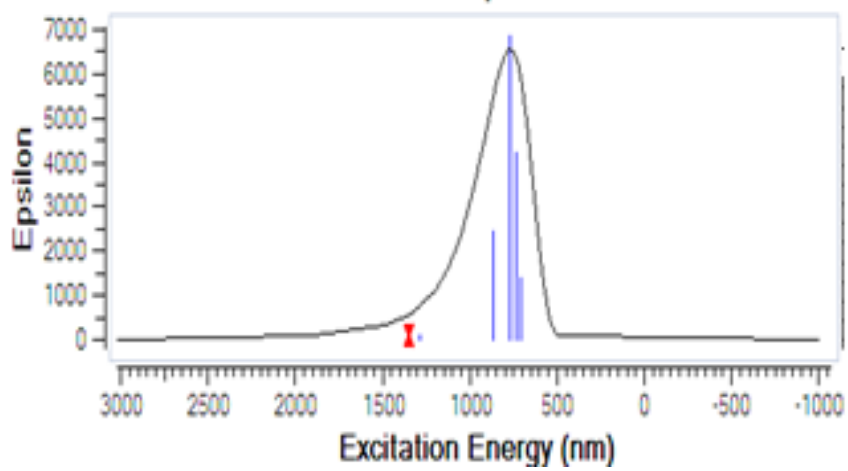

Figure (9). UV Visible spectrum of (a) AlP-diamantane, (b) Apraclonidine

\section{Conclusions}

1- AlP diamondoids are nano-particles with minimal surface effects, which make their electronic, structural properties as close as possible to those of bulk zincblende structure.

2- Diamondoids show minimal surface relaxation effects, which can be deduced from their bond lengths, tetrahedral angles and dihedral angles.

3- The bond lengths in AlP-diamantane found at $2.38 \AA$ and the experimental value of AlP bulk bond length at $2.293 \AA$.

4- Dihedral angles in AlP-diamantane the situation improves for the angles $-60^{\circ}$ and $60^{\circ}$ that become closer to its ideal value. Again, the dihedral values reflect the stability of diamondoids structures which is useful for our study to get the inert and no harm structures after bonding there with different drugs to use it as drugs carriers.

5- The energy levels are nearly discrete in AlP-diamantane.

6- The values of the energy gap diamantine is $(3.5 \mathrm{eV})$ with respect to the bulk value $(2.5 \mathrm{eV})$ as expected in this range this is agreement with all researches which tell us that the energy gap of nanomaterials is bigger than bulk of the same materials.

7- The green color signifies the neutral electrostatic potential. This means that diamantane insulating material and this helps us in the bonding process with the drug without that interact with any medication is delivery medicine to the affected places.

8- IR intensity of diamondoids divided into two regions depending on the properties of vibration or the gap separation them.

9- Raman spectrum of diamondoids Show the active region in IR is within less activity in Raman. 
10- UV Visible spectrum of diamondoids Excitation energy equal to (1.8849 $\mathrm{eV})$, the wave length Absorption is $(657.79 \mathrm{~nm})$ and frequency is $(\mathrm{f}=0.0002)$.

\section{Reference}

1- Van Zeghbroeck; B. J. "Bravais Lattices; Zincblende Lattice". University of Colorado, (1997).

2- Hamad R. Jappor, Zeyad Adnan Saleh, and Mudar A. Abdulsattar, "Simulation of Electronic Structure of Aluminum Phosphide Nanocrystals Using Ab Initio Large Unit Cell Method", Advances in Materials Science and Engineering, 6 Pages, Volume (2012).

3- G.Ali Mansoori, "Diamondoid Molecules" Advances in Chemical Physic Vol. 136, pp. 207-258, (2007).

4- Jacob Filik, "Diamondoid Hydrocarbons" chapter 1,p,126,Vol.65, (2010).

5- Robert A. Freitas Jr., "Meeting the Challenge of Building Diamondoid Medical Nanorobots", Institute for Molecular Manufacturing, May (2008).

6- Laboratories Alcon, S.S, F-68240 Kayersberg, France Revised: April (2004).

7- Robert A. Freitas Jr., "The Future of Aging”, Springer Science+Business Media B.V. ( 2010).

8- Wolfram Koch, Max C. Holthausen, “A Chemist's Guide to Density Functional Theory", Second EditionWiley-VCH Verlag GmbH, (Softcover); (Electronic) (2001).

9- Franci Bajd, "Van Der Waals Forces" university of Ljubljana Faculty of Mathematics and Physics April (2006).

10- NSM Archive, Physical Properties of Semiconductors, Accessed June 1, 2014. 11- Mudar Ahmed Abdulsattar, Bahjat B. Kadhimand Huda M. Jawad, "Electronic, Structural and Vibrational Properties of GaP Diamondoids and Nanocrystals: A Density Functional Theory Study" Nanomaterials and Nanotechnology Accepted 02 April (2015).

12- Nasir H N, Abdulsattar M A, Abduljalil H M, "Electronic Structure of Hydrogenated and Surface- Modified GaAs Nanocrystals: Ab Initio Calculations". Adv. Condens. Matter Phys., (2012).

13- Zhou A Q, O'Hern C S, Regan L, "The power of hard-sphere models: explaining

side-chain dihedral angle distributions of Thr" and Val. Biophys. J. 102: 2345, (2012).

14- Ali Taher Mohi Al-Sa'ady, "Density Functional and Semiempirical Investigations for Substituted Anthracene Molecules." A Thesis Submitted to the College of Science Al - Mustansiriyah University in Partial Fulfillment of the Requirements for the Degree of Doctor of Philosophy in Physics (2013). 